\title{
CASAS DE PARTO: SUA IMPORTÂNCIA NA HUMANIZAÇÃO DA ASSISTÊNCIA AO PARTO E NASCIMENTO
}

\author{
BIRTH CENTERS: IT'S IMPORTANCE IN THE HUMANIZATION OF DELIVERY AND BIRTH ASSISTANCE \\ CASAS DE PARTO: SU IMPORTANCIA EN LA HUMANIZACIÓN DE LA ASISTENCIA AL PARTO Y EL \\ NACIMIENTO
}

\section{Cleusa Alves Martins ${ }^{1}$, Nilza Alves Marques Almeida ${ }^{2}$, Maria Alves Barbosa ${ }^{3}$, Karina Machado Siqueira ${ }^{4}$, Karen Leverger Vasconcelos ${ }^{5}$, Marcus Antônio de Souza ${ }^{6}$, Tcherno Alliu Candé $^{7}$}

\begin{abstract}
RESUMO: Este trabalho apresenta reflexões sobre a importância do cuidar em obstetrícia, sobretudo a influência das Casas de Parto no sentido de nortear uma assistência humanizada e integral aos seus clientes. Objetiva discutir a importância da Casa de Parto na concretização do atendimento humanizado à parturiente. Salienta as deficiências do atual sistema de assistência à saúde da parturiente, que ainda se caracteriza por concepções do modelo biomédico e condutas que, muitas vezes, expõe a cliente a situações de desrespeito e exclusão. Propõe-se a assistência de Enfermagem respaldada em pilares como o conhecimento técnico-científico e habilidades nas relações intra e interpessoais, reverenciando o cuidado em saúde como norteador das condutas do profissional. Enfatiza-se a necessidade de buscar a assistência humanizada à mulher no ciclo gravídicopuerperal, tornando-a, por meio de um processo saudável de comunicação, a protagonista do evento que envolve atendimento integral obstétrico e neonatal. Foram definidas duas categorias de análises: Casas de Parto e Humanização; O Cuidar no Processo do Nascimento. Concluiu-se que a Casa de Parto apresenta como uma opção adequada que proporcione assistência humanizada à parturiente e familiares, e sua concretização caminha, também, por mudança de postura do profissional de saúde.
\end{abstract}

PALAVRAS CHAVE: Humanização do Parto; Enfermagem Obstétrica; Centros Independentes de Assistência a Gravidez e ao Parto.

SUMMARY: This article presents reflections about the importance of care in obstetrics, above all about the infuence of Birth Centers on the way of whole and humanizing assistance for their clients. It aims to discuss the importance of Birth Centers to render concrete a humanizing assitance for the parturient. It points out the deficiency of the actual health assistance system to the parturient that still is caracterized by conceptions of the biomedical model besides conducts that, many times, exposes the client to situations of disrespect and exclusion. It proposes nursing assistance based on pillars such as scientific and technical knowledge and skills in the relations between the person and the self and with the others, honoring health care as a guideline of professionals' conducts. The need to search for a humanizing assistance to women in puerperal-pregnancy cycle is emphasized, transforming them in the protagonist of the event that involves full obstetrics and neonatal assitance, using means like a healthy communication process. Two categories of analysis were defined: Birth Centers and Humanization; Care in Birth Process. It was concluded that Birth Centers presents a proper option that provides a humanizing assitance for the parturient and her family and that its concretization depends on the modification of the posture of health professionals.

KEY WORDS: Humanizing Delivery; Obstetrical Nursing; Birthing Centers.

Enfermeira. Doutora em Enfermagem. Professor Adjunto da Faculdade de Enfermagem da Universidade Federal de Goiás. Rua 227, Qd 68 s/n (FEN/UFG); Setor Leste Universitário, CEP 74605080, Goiânia - Goiás. E-mail: cleusa@fen.ufg.br

${ }^{2}$ Enfermeira. Mestre em Biologia. Doutoranda no Programa de Mestrado e Doutorado em Ciências da Saúde - Convênio Multiinstitucional (UnB/UFG/UFMS). Professor Assistente da Faculdade de Enfermagem da Universidade Federal de Goiás. Email: nilza@fen.ufg.br

${ }^{3}$ Enfermeira. Doutora em Enfermagem. Professora Titular da Faculdade de Enfermagem da Universidade Federal de Goiás. Email: mbarbosa@fen.ufg.br

${ }^{4}$ Enfermeira. Mestranda do Programa de Pós-graduação Stricto Sensu da Faculdade de Enfermagem da Universidade Federal de Goiás. Bolsista CNPq E-mail: karinamsiqueira@hotmail.com

${ }^{5}$ Enfermeira Obstétrica, Professor Auxiliar da Disciplina Obstétrica da Faculdade de Enfermagem da Universidade Federal de Goiás FEN/UFG

${ }^{6}$ Acadêmico de Graduação em Enfermagem. Faculdade de Enfermagem da Universidade Federal de Goiás (FEN/UFG). marcus souza@pop.com.br

${ }^{7}$ Acadêmico de Graduação em Enfermagem. Faculdade de Enfermagem da Universidade Federal de Goiás. tchernoa@hotmail.com 
RESUMEN: Este trabajo presenta reflexiones sobre la importancia del cuidar en obstetricia, sobre todo la influencia de las Casa de Parto en el sentido de nortear una asistencia humanizada e integral a sus clientes.El objetivo es discutir la importancia de la Casa de Parto en la concretización de la atención humanizada a la parturiente. Se destacan las deficiencias del actual sistema de asistencia a la salud de la parturiente, que todavía se caracteriza por concepciones del modelo biomédico y conductas que, muchas veces, expone al cliente a situaciones de falta de respeto y exclusión.Se propone la asistencia de Enfermería respaldada en pilares como el conocimiento técnico- científico y habilidades en las relaciones intra e inter-personales, reverenciando el cuidado en salud como norteador de las conductas del profesional. Se enfatiza la necesidad

\section{INTRODUÇÃO}

Na garantia da perpetuação da espécie, encerra o sentido biológico da luta da raça humana pela sobrevivência, desde a possibilidade de sua existência. Nessa sucessão de gerações escrevemos a nossa história, construímos a nossa cultura, adotamos, abandonamos e substituímos normas, valores, posturas e condutas.

Nos diferentes modos que os povos tenham se organizado, o aspecto reprodutivo sempre teve seu destaque, reservando à mulher a sua vital participação como garantia da continuidade da linhagem, a garantia da família, a célula-máter da sociedade.

Entre os astecas, a mulher era cuidada carinhosamente durante a gestação e o parto com cerimônias que humanizavam a atenção: a futura mãe devia alimentar-se bem, a parteira dava conselhos para o bom desenvolvimento do filho e recomendava relações sexuais nos primeiros meses de gravidez com o objetivo de fortalecer e fazer crescer o produto (REZENDE, 2002, p. 278).

Porém, fenômenos naturais, como, por exemplo, a reprodução humana, vêm sofrendo drásticas interferências em sua essência. Pois, os povos se envolvem em um modelo estruturado na racionalidade, no tecnicismo, mercantilismo e na carência de princípios humanísticos, onde mãe e filho são vistos apenas sujeitos da assistência, negligenciando sua integralidade como pessoas (CARON \& SILVA, 2002).

O modelo cartesiano esteve intimamente ligado ao desenvolvimento histórico da biologia, mostra-se uma associação com a medicina, desde Hipócrates e Galeno, que contribuíram decisivamente para o conhecimento no campo biológico, a partir de então, outros nomes famosos prosseguiram com fracassos e êxitos estendendo-se pelo século XVII.

René Descartes criou uma imagem inflexível dos organismos vivos como sistemas mecânicos e, desse modo estabeleceu uma rígida estrutura conceitual, construindo novos caminhos da fisiologia para seus de buscar la asistencia humanizada a la mujer en el ciclo embarazo-puerperal, consiguiendo, por medio de un proceso saludable de comunicación, la protagonista del evento que envuelve la atención integral obstétrica y neonatal. Fueron definidas dos categorías de análisis: Casas de Parto y Humanización; El Cuidar en el Proceso del Nacimiento. Se concluye que la Casa de Parto puede ser una opción adecuada que proporcione una atención humanizada a la parturiente y familiares, y su concretización camina, también, por cambios de postura del profesional de salud.

PALABRAS CLAVE: Humanización del Parto; Enfermería Obstétrica; Centros Independientes de Asistencia al Embarazo y al Parto.

seguidores com a tarefa de elaborar os detalhes da concepção mecanicista da vida. Nesse sentido, o primeiro a obter êxito foi Giovanni Borello, um discípulo de Galileu, que conseguiu explicar aspectos básicos da ação muscular em termos mecanicista. Mas o grande sucesso da fisiologia ocorreu quando William Harvey que aplicou o modelo mecanicista do fenômeno da circulação sanguínea. Inspirando outros fisiologistas de seu tempo a aplicar o método em descrições de outras funções corporais. (CAPRA, 1982, p. 99).

Destaca-se, ainda, Isaac Newton que revolucionou com sua física clássica por meio da comprovação matemática de muitas teorias, como as de Galileu Galilei que deram o respaldo científico a uma série de inovações e avanços tecnológicos nas mais diversas áreas; Albert Einsten com a física moderna desvendou os segredos sutis e elementares da matéria que nos cerca, dando à ciência a direção dos passos a serem pautados, numa velocidade vertiginosa, desafiando as possibilidades de raciocínio e imaginação do ser humano rumo à revolução tecnológica, à robotização, à automatização, à informatização.

O tratamento de importantes doenças provém, essencialmente, de uma abordagem reducionista, altamente mecanizada, especializada e impessoal, de um modelo exíguo para explicar enfoques mais abrangentes como a saúde da população.

Essas descobertas representam papel decisivo no avanço da assistência ao ser humano, no entanto, necessário se faz, transcender o modelo biomédico e discutir a redefinição da aplicação tecnológica na questão da saúde, sob o prisma da enfermagem como prática assistencial humanizada, integral, à saúde da mulher.

Embora os profissionais disponham de considerável poder de mudanças, no sistema de saúde a manifestação mais poderosa é determinada pela supremacia do poder econômico. E, a política de saúde brasileira, não é diferente dos países em 
MARTINS, Cleusa Alves; ALMEIDA, Nilza Alves Marques; SOUZA, Marcus Antônio, CANDÉ, TCHERNO; SIQUEIRA, Karina Machado, BARBOSA, Maria Alves. CASAS DE PARTO: SUA IMPORTÂNCIA NA HUMANIZAÇÃO DA ASSISTÊNCIA AO PARTO E NASCIMENTO. Revista Eletrônica de Enfermagem, v. 07, n. 03, p. 360 - 365, 2005. Disponível em http://www.fen.ufg.br/Revista/revista7 3/revisao 03.htm

desenvolvimento, portanto, sofre influência direta da política sócio-econômica determinada por organismos internacionais - como o Fundo Monetário Internacional (FMI), o Banco Mundial (BM), e supranacionais como a Organização Mundial de Saúde (OMS) e a Organização das Nações Unidas para a Educação, a Ciência e a Cultura (UNESCO), no que diz respeito, a gastos internos, financiamento de projetos, dentre outras exigências do sistema tecnológico industrial.

Partindo para a instituição saúde, nos dias de hoje, deparamos com uma prática assistencial humanística decorrente de conquista dos movimentos populares. Ainda assim, no cotidiano dos serviços de saúde na área obstétrica - pré-natal, trabalho de parto, parto, puerpério - existe uma demanda para conseguir a assistência pré-natal e, muitas vezes, nas consultas as queixas e dúvidas das gestantes são pouco valorizadas. Recebem informações que se equivalem a um verdadeiro compêndio de proibições, recomendações, restrições e exigências e voltam para suas casas com a cabeça em verdadeiro conflito, sem saber se estão grávidas ou doentes.

Próximo a data provável do parto, ao sentirem as primeiras contrações, as gestantes percorrem mais uma etapa de sua peregrinação, vias-sacras, procuram a rede de saúde, sendo assim, as próprias vítimas da exclusão social da mulher.

Complicações na gestação, no parto e no puerpério aparecem com destaque como uma das dez primeiras causas de morte de mulheres. Segundo o Ministério da Saúde, 92\% desses casos poderiam ser evitados. (BRASIL, 2005a).

Para nós, a mulher no ciclo gravídico-puerperal se encontra inserida em seu meio sócio-econômico e cultural, do qual traz uma bagagem espiritual e emocional, medos, preconceitos, vontades; podem estar trazendo decepções, dissabores, mágoas, tristezas e também alegrias, felicidades, ânimo, esperança, fé, necessitando assim, ser atendida em sua integralidade, pois, não há, na vida da mulher, fase tão repleta de dúvidas, ansiedades e frustrações como o ciclo grávido-puerperal, a demonstrar a íntima relação entre os fenômenos psíquicos e os somáticos (REZENDE, 2002, p. 278).

A institucionalização da assistência humanizada à mulher, preconizada pelo Ministério da Saúde visa garantir a gestante o acesso ao atendimento digno e de qualidade no decorrer da gestação, parto e puerpério (BRASIL, 2000).

A partir da necessidade de estabelecer novo paradigma assistencial, na assistência obstétrica e perinatal, em junho de 2000, o Ministério da Saúde instituiu o Programa de Humanização no Pré-Natal, Parto e Nascimento - PHPN (SERRUYA et. al. 2004). Esse programa é importante instrumento para a organização e estruturação do atendimento à grande demanda de gestantes por: "centralizar esforços no sentido de reduzir as altas taxas de morbi-mortalidade materna e perinatal; adotar medidas que assegurem a melhoria do acesso, da cobertura e da qualidade do acompanhamento pré-natal, da assistência ao parto, puerpério e neonatal; e ampliar as ações já adotadas pelo Ministério da Saúde na área de atenção à gestante, com investimentos nas redes estaduais de assistência à gestação de alto risco, o incremento do custeio de procedimentos específicos e outras ações, como o Projeto de Capacitação de Parteiras Tradicionais, do financiamento de cursos de especialização em enfermagem obstétrica e a realização de investimentos nas unidades hospitalares integrantes destas redes." (BRASIL, 2000).

No propósito de ampliar a rede asistencial, em 1998, foi inaugurada pela Fundação Zerbini do Instituto do coração da Faculdade de Medicina da Universidade de São Paulo, a primeira Casa de Parto vinculada ao projeto Qualidade Integral em Saúde (Qualis) do Programa Saúde da Família (SEPÙLVEDA, 2000 apud HOGA, 2004b).

A criação de Casas de Parto para o atendimento das parturientes não acometidas por doenças clínicas e ou obstétricas foi proposta porque se acreditou que esta seria uma alternativa possível à humanização da assistência nesse âmbito (HOGA, 2004a).

Nesta perspectiva, as Casas de Parto visam a valorizar o fenômeno do parto e nascimento em sua essência fisiobiológica como um processo natural e na sua dimensão social. Funciona como uma unidade autônoma, dispondo de recursos materiais e humanos compatíveis para prestar assistência de qualidade às gestantes, parturiente e aos nascituros. Geralmente, é construída em convênio com unidades de referências, próxima ao hospital ou maternidade e, constituída por equipe mínima: um enfermeiro obstetra, um auxiliar de enfermagem, um auxiliar de serviços gerais e um motorista de ambulância (HADDAD, 2004).

Nesta perspectiva, buscando algumas reflexões sobre as casas de parto e sua importância na assistência de enfermagem ao parto e ao nascimento, com base na literatura publicada em periódicos da área de Enfermagem e de Obstetrícia, tais como a Revista Latino-americana de Enfermagem, Revista Eletrônica de Enfermagem, Revista Brasileira de Enfermagem, Revista Mineira de Enfermagem e Revista Brasileira de Ginecologia e Obstetrícia. A partir dos textos identificados, traçamos tais reflexões a partir de três eixos que apresentamos a seguir.

\section{O CUIDAR NO PROCESSO DO NASCIMENTO}

Em alguns países do mundo - Japão, Holanda, Inglaterra e outros, diferentemente do Brasil, o parto é visto de forma natural e simples, normalmente, apresentando menores taxas de mortalidade materna e complicações no pós-parto (BRASIL, 2005b). 
MARTINS, Cleusa Alves; ALMEIDA, Nilza Alves Marques; SOUZA, Marcus Antônio, CANDÉ, TCHERNO; SIQUEIRA, Karina Machado, BARBOSA, Maria Alves. CASAS DE PARTO: SUA IMPORTÂNCIA NA HUMANIZAÇÃO DA ASSISTÊNCIA AO PARTO E NASCIMENTO. Revista Eletrônica de Enfermagem, v. 07, n. 03, p. 360 - 365, 2005. Disponível em http://www.fen.ufg.br/Revista/revista7 3/revisao 03.htm

O parto é um acontecimento único, de inestimável importância, uma experiência ímpar que envolve o seu parceiro, suas famílias e a comunidade a qual a gestante faz parte.

"(...) é considerado como um processo psicossomático, onde o comportamento da mulher vai depender, além da própria evolução do trabalho de parto, do nível de informação da mulher, a sua historia pessoal, contexto sócioeconômico, personalidade e simbolismo" (DAVIM \& MENEZES, 2001, p. 64).

O parto e nascimento é um mecanismo fisiológico, e influenciado por uma série de fatores como hormônios, estado orgânico e psico-emocional da mulher. Mas, na contra-mão desse processo nos deparamos com a institucionalização da assistência de forma impessoal, mecanicista e alopática com procedimentos invasores bombardeando-o com medicamentos como se estivesse tratando uma ameaça à espécie humana.

A obstetrícia moderna nasceu sob a tutela da cirurgia e transformou o parto e o nascimento em um evento medicalizado, descaracterizando a essência original de fenômeno existencial e psicológico, para mãe e filho, e acontecimento social, para o grupo familiar e sociedade. (CARON \& SILVA, 2002, p. 486).

A mulher vivencia o parto com o significado de dor, desespero, e abandono, neste contexto, a assistência busca recursos em medicamentos para atenuar o sofrimento. Muitos profissionais consideram a dor, a ansiedade e o medo como outros fatores relevantes durante o trabalho de parto (ALMEIDA et al, 2005).

Essa conduta, simplesmente, se torna cada vez mais inapropriada, e evidencia a ilegitimidade da essência humana, no fenômeno do parto e nascimento de uma criança.

A Organização Mundial de Saúde preconiza que a mulher em trabalho de parto deve ter suporte emocional e atenção à saúde. Porém, longe de vivenciar tais recomendações, o modelo assistencial de saúde adotado negligencia os benefícios advindos do parto normal, sem dizer das condições trágicas que se dão os partos, atualmente, no Brasil (DAVIM \& MENEZES, 2001).

$\mathrm{Na}$ realidade cotidiana, ainda se depara com profissionais que exigem da gestante um comportamento passivo de aceitação da conduta. Percebe-se que, além do conhecimento técnicocientífico que detém, não vivenciam a sensibilidade, a paciência, a compreensão, enfim, habilidades que não Ihes foram anteriormente despertadas.

Fatores como estes tornam o atendimento tão impessoal e podem contribuir, de certa forma, para o surgimento de complicações no trabalho de parto como as distócias funcionais e feto-anexiais (DAVIM \& MENEZES, 2001).

Ignora-se que o cuidado humano deve ocorrer dentro do respeito à dignidade, à sensibilidade para como o sofrimento, bem como na ajuda para superálo, para enfrentá-lo e para aceitar o inevitável (WALDOW, 2001, p.14).

Humanizar a parturição é envolver-se com-ooutro; é lembrar que no momento do parto está ocorrendo a separação de dois corpos, que até esse momento viveram juntos, um dentro do outro, em ralação de dependência e de íntimo contato. Nesse momento o enfermeiro necessita restaurar a tranqüilidade, o equilíbrio, a harmonia interior, com o cuidado, porque é por ele que exercitamos o encontro do ser profissional com 0 ser-parturiente, restabelecendo o sentimento de perda e esvaziamento, que a parturiente vivencia de forma tão intensa e particular (ZAGONEL, 1997, p.36).

Não é possível falar de assistência no ciclo gravídico-puerperal sem reverenciarmos o cuidar:

O cuidado por sua própria natureza possui dois significados que se inter-relacionam, por ser uma atitude de atenção e solicitude para com o outro, ao mesmo tempo em que representa preocupação e inquietação, pois o cuidador se sente envolvido afetivamente e ligado ao outro (DAMAS et. al., 2004).

MARTINS E PEREIRA (2002) em seu estudo de avaliação da qualidade da assistência obstétrica e perinatal afirmam que a nomatização da assistência humanizada ao parto e nascimento, na instituição pesquisa, foi importante para evitar práticas consideradas inapropriadas, segundo relatos de depoentes - é importante olhar para o casal grávido e tentar entender o significado da gravidez para os pais. Pois nós somos o cartão de visita, a comissão de frente faz a festa...existe grande interação entre equipe de saúde e usuárias. Relatam, ainda que essa abordagem facilita a interação e fortalecimento dos laços afetivos mãe-pai-filho e conseqüentemente o sucesso do aleitamento materno.

Entendemos o cuidado como um processo de ser-estar por parte do cuidador a quem será cuidado, que envolve habilidades técnicas, conhecimento, sensibilidade, solidariedade, carinho, amor, consideração. Portanto torna-se desnecessário e redundante falar em cuidado humanizado, pois entendemos que cuidado é o que confere a condição de humanidade às pessoas (WALDOW, 2004, p. 37).

Nesse paradigma de cuidado, a sua importância possui reflexão muito significativa durante o parto normal e/ou fisiológico, onde além do temor inerente ao parto, a mulher sente medo de quem a atenderá, por isso, seria indispensável o estabelecimento de uma comunicação efetiva com o cliente, para a construção de uma relação terapêutica que estabelecerá uma condução de trabalho de parto resolutiva e não intervencionista (CARON \& SILVA, 2005).

A enfermagem tem por ofício e filosofia de atuação, estudo e pesquisas que visem: 
“(...) exercitar o cuidado em sua plenitude, de forma integral, em uma visão mais humanista que apresente um toque diferenciado ao ser visualizado como um comportamento interativo, como uma expressão de ser-aí-no mundo, com base em princípios morais, respeitando e considerando o ser humano em sua totalidade" (WALDOW, 2004, p.12)

\section{CASAS DE PARTO E HUMANIZAÇÃO}

A atuação do profissional de enfermagem deve pautar, por essência, o cuidar. Pois, "encontra-se na raiz primeira do ser humano, antes que ele faça qualquer coisa". (BOFF, 2003, p.34).

Diante dos desafios a serem vencidos, referentes ao parto humanizado, as Casas de Parto apontam como alternativa viável que proporciona uma assistência humanizada à mulher no ciclo gravídicopuerperal. Trata-se de uma estrutura institucional menor, preconiza a atuação autônoma do enfermeiro obstétrico, ou seja: facilita a integração da equipe de trabalho, possibilita a colaboração e respeito mútuo entre o grupo profissional e os clientes (HOGA, 2004a).

A Casa de Parto é uma unidade de saúde destinada à assistência humanizada e de qualidade ao parto fisiológico, é a representação simbólica de um conjunto de idéias guiadas por um paradigma próprio e diferente do vigente no sistema de saúde atual. E, também, é o símbolo da necessária transformação da cultura predominante nas instituições que prestam assistência ao parto e nascimento (HOGA, 2004b).

Para o Ministério da Saúde, dois aspectos são fundamentais na humanização: o primeiro diz respeito à convicção de que é dever das unidades de saúde receber com dignidade a mulher, seus familiares e o recém-nascido. O segundo se refere à adoção de medidas e procedimentos sabidamente benéficos para o acompanhamento do parto e do nascimento, evitar práticas intervencionistas desnecessárias, tradicionalmente realizadas, e que não beneficiam a mulher nem o recém-nascido e que, com freqüência, acarretam maiores riscos para ambos. Receber com dignidade a mulher requer atitude ética e solidária por parte dos profissionais de saúde.

Neste sentido, a Casa de Parto é humanizar, é dar a voz da parturiente, permitir que ela seja consciente da sua posição como figura central no processo, fazendo valer seus direitos, sua autonomia e seu valor pessoal. E, cabe ao profissional que almeja uma atuação humanista, promover o envolvimento efetivo da parturiente neste processo como sujeito ativo e consciente dos seus direitos.

\section{CONSIDERAÇÕES FINAIS}

O respeito que os profissionais de saúde estabelecem com as mulheres e familiares, durante no processo de parturição, é condição indispensável para humanização da assistência ao parto.

Imbuídos deste ideal, os profissionais de saúde necessitam aproveitar todas as possibilidades e oportunidades de serem sensíveis, honestos e competentes, pois o tempo passa e nos surpreende com os resultados de nossas ações.

O graduando de enfermagem, por sua vez, recebe um direcionamento da atenção integral da prática do cuidar, desempenha atividades de interesse individual e grupal e apreende condutas condizentes com essa abordagem, para entender as necessidades e limites do outro, ser humano.

A Casa de Parto pode ser uma opção apropriada para se materializar o cuidado integral à mulher parturiente e seu familiar. A instituição e a postura ética do cuidador permeiam a filosofia e os princípios que estabelecem a interação individualizada, coloca as clientes como sujeitos ativos do processo de parturição, respeita os seus direitos de cidadãs, preconizados pela Constituição brasileira.

Por fim, a humanização da assistência obstétrica e neonatal, ainda depende muito do modo de ser do profissional de saúde, pois apenas as prerrogativas institucionalizadas não são suficientes para adoção de novos paradigmas. Estudos e pesquisas na área revelam que em relação ao impacto social, principalmente, 0 atendimento causa satisfação na clientela, evidencia-se uma presença ainda concreta na nova abordagem assistencial, dentro dos conteúdos da temática humanista.

\section{REFERÊNCIAS BIBLIOGRÁFICAS}

ALMEIDA et al. Utilização de técnicas de respiração e relaxamento para alivio de dor e ansiedade no processo de parturição. Revista Latino-Americana de Enfermagem, v.13, n.1, p 52-58, 2005,

BOFF, L. Saber cuidar: ética do humanocompaixão pela terra. Petrópolis: Vozes, 2003.

BRASIL, Ministério da Saúde. Programa de Humanização no Pré-natal e Nascimento, 2000 [online]. Disponível em: http://dtr2001.saude.gov.br/sps/areastecnicas/m ulher/Cartilha.htm [Acesso em 21 jul. 2005].

BRASIL, Ministério da Saúde. Mulheres precisam ter mais cuidado com a saúde. 2005a [online]. Disponível em: http://www.universodamulher.com.br/index.php? mod=mat\&id materia=4264 [Acesso em 25 jul. 2005].

BRASIL, Ministério da Saúde. Amigas do Parto 2005b [online]. Disponível em: http://www.amigasdoparto.com.br/phoje.html. [Acesso em 25 jul. 2005b]. 
CAPRA, F. O ponto de mutação. Trad. Álvaro Cabral. São Paulo: Cultrix, 1982.

CARON, O. A. F.; SILVA, I. A. Parturiente e Equipe Obstétrica: a difícil arte da comunicação. Rev. Latino-Am. Enf., v.10 n.4, p. 485-492. 2002.

DAMAS, K. C. A.; MUNARI, D. B.; SIQUEIRA, K. M. Cuidando do cuidador: reflexões sobre o aprendizado dessa habilidade. Revista Eletrônica. de Enfermagem, v. 06, n. 02, 2004 [online]. Disponível em: www.fen.ufg.br/revista/revista6_2/cuidador.html [Acesso em 05 jul. 2005].

DAVIM, R. M. B.; MENEZES, R. M. P.; Assistência ao parto normal no domicílio. Rev. Latino-Am. Enf. v.9, n.6, p. 62-68. 2001.

FIGUEREDO, et al. Indicadores de cuidados para o corpo que pro-cria: ações de enfermagem no pré-natal e pós-natal - uma contribuição para a prática de enfermagem. Rev. Latino-Am. Enf., v.12, n. 6, p. 905-912. 2004.

HADDAD E. B. Revogação da Liminar em Desfavor à Casa de Parto no Rio de Janeiro Casas de Parto no Mundo. 2004 [online]. Disponível em: http://www.casasdeparto.com.br/casasdeparto/r evogacao.asp [Acesso em 25 jul. 2005].

HOGA, L. A. K. Casas de Parto: simbologia e princípios assistenciais. Rev.Bras.Enf., v. 57, n. 5, p. 537-540, 2004.

Motivação das enfermeiras obstétricas para o desenvolvimento de seu trabalho em uma casa de parto. Rev. Min.Enf., v. 8, n. 3, p. 368-372. 2004.

MARTINS, C. A. e PEREIRA, S. V. M. Qualidade da assistência obstétrica e perinatal em hospital amigo da criança: experiência de maternidade - Anápolis/GO. Rev. Anna Nery, v. 6, n. 3, p. 451-463. 2002.

REZENDE, J. Obstetrícia. Rio de Janeiro: Guanabara Koogan. 2002.

SERRUYA, S. J.; LAGO, T. G.; CECATTI, G. J. Avaliação preliminar do programa de humanização no pré-natal e nascimento no Brasil. Rev.Bras. Ginec.Obst., v. 26 n.7, p. 517525. 2004.

SILVA, M. J. P. (org.) Qual o tempo do cuidado? Humanizando os cuidados de Enfermagem. São Paulo: Loyola, 2004.

WALDOW, V. R. O cuidado na saúde as relações entre o eu, o outro e o cosmos. Petrópolis: Vozes, 2004.

WALDOW, V.R. Cuidado humano o resgate necessário. Porto Alegre: Editora Sagra Luzzatto. 2001.

ZAGONEL, I. P. S., Contribuição do cuidado de enfermagem à humanização da parturição. Cogit.Enf., v. 2, n.2, p.34-8. 1997.
Texto Recebido em 25/09/2005

Publicação Aprovada em 29/12/2005 